\title{
Evaluation of Emergency Department Data Quality following PHIN Syndromic Surveillance Messaging Guide
}

\author{
Hwa-Gan Chang ${ }^{\star 1}$, Charlene Weng ${ }^{1}$, Charlie DiDonato ${ }^{2}$, Dave DiCesare ${ }^{1}$, Jian-Hua Chen ${ }^{1}$ \\ and Debra Blog 1
}

${ }^{1} \mathrm{NYSDOH}$, Albany, NY, USA; ${ }^{2} \mathrm{NTT}$ Data, Albany, NY, USA

\section{Objective}

To evaluate the readiness and timeliness of ED data submitted by hospitals following PHIN syndromic surveillance messaging guide and to evaluate the availability of minimum data elements. To validate the accuracy and completeness of data from ADT messages compared with data currently reported to the NY syndromic surveillance system.

\section{Introduction}

The final rules released by the Centers for Medicare and Medicaid Services specified the initial criteria for eligible hospitals to qualify for an incentive payment by demonstrating meaningful use of certified Electronic Health Record (EHR) technology. Syndromic surveillance reporting is one of three public health objectives that eligible hospitals can choose for stage 1 . The PHIN messaging guide for syndromic surveillance was published for hospitals to construct emergency department data using Admit Discharge Transfer (ADT) messages, with the minimum dataset that is standard among hospitals and public health agencies.

Currently New York hospitals are reporting emergency department (ED) visit data to the NY syndromic surveillance (SS) system. Patient chief complaint data are monitored for trends of illness at the community level in order to detect possible outbreaks and situational awareness.

\section{Methods}

12 hospitals using three EHR certified vendors pilot tested syndromic surveillance data for MU. Hospitals started to transmit ED data in HL7 v 2.5.1 to the NY pre-certification server beginning October 2011. The month of data from July 2012 was evaluated for availability by data elements listed in the implementation guide. The ADT message types were analyzed and the timeliness of reporting was calculated from visit date to report date of the first message type. The data from the pre-certification server was matched against data from the production SS system by medical record number and visit date to evaluate the data content.

\section{Results}

There were 5 hospitals from vendor A, 3 from vendor B and 4 from vendor $\mathrm{C}$ participating in the pilot testing; 5854, 9882, and $13316 \mathrm{ED}$ visits were reported from the three vendors respectively for the month of July. The type of first message by vendor is shown in Table 1 . The availability of data elements is listed in Table 2 . There were $79 \%$, $82 \%$, and $87 \%$ ED visit records received within 24 hours for vendor $\mathrm{A}, \mathrm{B}$, and $\mathrm{C}$ respectively. One hospital from vendor A, 3 hospitals from vendor $\mathrm{B}$ and 4 hospitals from vendor $\mathrm{C}$ also reported $\mathrm{ED}$ data to the production system, and their comparison with pilot testing data is shown in Table 3.

\section{Conclusions}

The types of ADT messages first reported varied by vendor and hospital. Not all data elements specified in the implementation guide were available or complete, and varied by vendor. An average $83 \%$ of first messages were received within 24 hours and the chief complaint from ADT messages did not match well with the current ED system in production. It is a very time consuming and resource demanding process to move a hospital from successful attestation stage to production and requires public health, EHR vendor, and hospital IT to work together. The learning experience from these three vendors in implementing syndromic surveillance for MU will help public health and EHR vendors to prepare for stage 2 .

Table 1: Type of First ADT Message by Vendor

\begin{tabular}{|c|c|c|c|}
\hline ADT Message & $\begin{array}{c}\text { Vendor A } \\
\mathrm{N}(\%)\end{array}$ & $\begin{array}{c}\text { Vendor B } \\
\mathrm{N}(\%)\end{array}$ & $\begin{array}{c}\text { Vendor C } \\
\mathrm{N}(\%)\end{array}$ \\
\hline A01 & $2543(43 \%)$ & 43 & $198(2 \%)$ \\
\hline A04 & $2757(47 \%)$ & 0 & $12396(93 \%)$ \\
\hline A08 & $553(10 \%)$ & $1635(17 \%)$ & $686(5 \%)$ \\
\hline A03 & 0 & $8204(83 \%)$ & 8 \\
\hline total & 5854 & 9882 & 13316 \\
\hline
\end{tabular}

Table 3: Percent Matched Data Content by Vendor

\begin{tabular}{|c|c|c|c|}
\hline Data Element & $\begin{array}{c}\text { Vendor A } \\
\%\end{array}$ & $\begin{array}{c}\text { Vendor B } \\
\%\end{array}$ & $\begin{array}{c}\text { Vendor C } \\
\%\end{array}$ \\
\hline Date of birth & $99 \%$ & $74 \%$ & $100 \%$ \\
\hline Zipcode & $93 \%$ & $99 \%$ & $99 \%$ \\
\hline Sex & $99 \%$ & $100 \%$ & $100 \%$ \\
\hline Discharge disposition & $52 \%$ & $65 \%$ & $75 \%$ \\
\hline Chief complaint & $19 \%$ & $0 \%$ & $83 \%$ \\
\hline
\end{tabular}

Table 2: Data Element Availability by Vendor

\begin{tabular}{|c|c|c|c|}
\hline MDS data elements availability & Vendor A & Vendor B & Vendor C \\
\hline Date of birth & $100 \%$ & $100 \%$ & $100 \%$ \\
\hline Gender & $100 \%$ & $100 \%$ & $100 \%$ \\
\hline Zipcode & $100 \%$ & $100 \%$ & $100 \%$ \\
\hline Race & $96 \%$ & $99 \%$ & $97 \%$ \\
\hline Ethnicity & $100 \%$ & $0 \%$ & $87 \%$ \\
\hline Symptoms Onset Date & $0 \%$ & $0 \%$ & $0 \%$ \\
\hline Chief Complaint: text & $80 \%$ & $95 \%$ & $91 \%$ \\
\hline Admit Reason & $72 \%$ & $58 \%$ & $91 \%$ \\
\hline Diagnosis Code & $0 \%$ & $4 \%$ & $14 \%$ \\
\hline Discharge Disposition & $50 \%$ & $95 \%$ & $90 \%$ \\
\hline Temperature & $0 \%$ & $3 \%$ & $24 \%$ \\
\hline Pulse Oximetry & $0 \%$ & $0 \%$ & $24 \%$ \\
\hline
\end{tabular}

\section{Keywords}

Syndromic surveillance; Meaningful Use; PHIN messaging guide

*Hwa-Gan Chang

E-mail: hgc04@health.state.ny.us 